\title{
A Case Study for Improving a Collaborative Design Process
}

Sophie Dupuy-Chessa, Nadine Mandran, Guillaume Godet-Bar, and Dominique Rieu

\author{
University of Grenoble, CNRS, LIG \\ 385 rue de la Bibliothèque \\ 38041 Grenoble Cedex 9, France \\ Firstname. Lastnamedimag.fr
}

\begin{abstract}
We propose a design method for supporting the design of rich user interfaces. It integrates software engineering and human-computer interaction practices through collaborations and focuses on the traceability of processes and models. In this paper, we investigate these collaborative aspects with a case study, which gave us some insights in order to improve the process.
\end{abstract}

Keywords: Collaboration, Process, Qualitative study.

\section{Introduction}

The Software Engineering (SE) methods have shown their reliability for specifying and developing the functional core of information systems. Nowadays, such systems can have rich user interfaces based on interaction techniques like vocal commands or gesture recognition. To guide their design, we propose the Extended Symphony method [1]. It has been designed for facilitating collaborations between SE and Human Computer Interaction (HCI) specialists and for enabling designers to develop rich user interfaces. But it was still a theoretical proposal that needed to be confronted to practical issues. Then we realized empirical studies focused on specific parts of the method [2]. One of them is presented in this article. It gave us some insights about the collaborative aspects of the method. It studied two hypotheses made while designing the extension of Symphony: 1) the process facilitates the collaboration between actors from the SE and HCI domains and 2) it allows designers to produce consistent models.

The following section gives an overview of the collaborative process of the Extended Symphony method. Then we present the case study, before concluding with some perspectives.

\section{The Extended Symphony Method}

Originally developed by the UMANIS Company, Symphony is based on a Y-shaped development cycle whose functional (left) branch corresponds to the traditional task of domain modelling, independently from technical aspects. This branch whose an excerpt is given in Fig. 1, focuses on the integration of SE and HCI practices. 


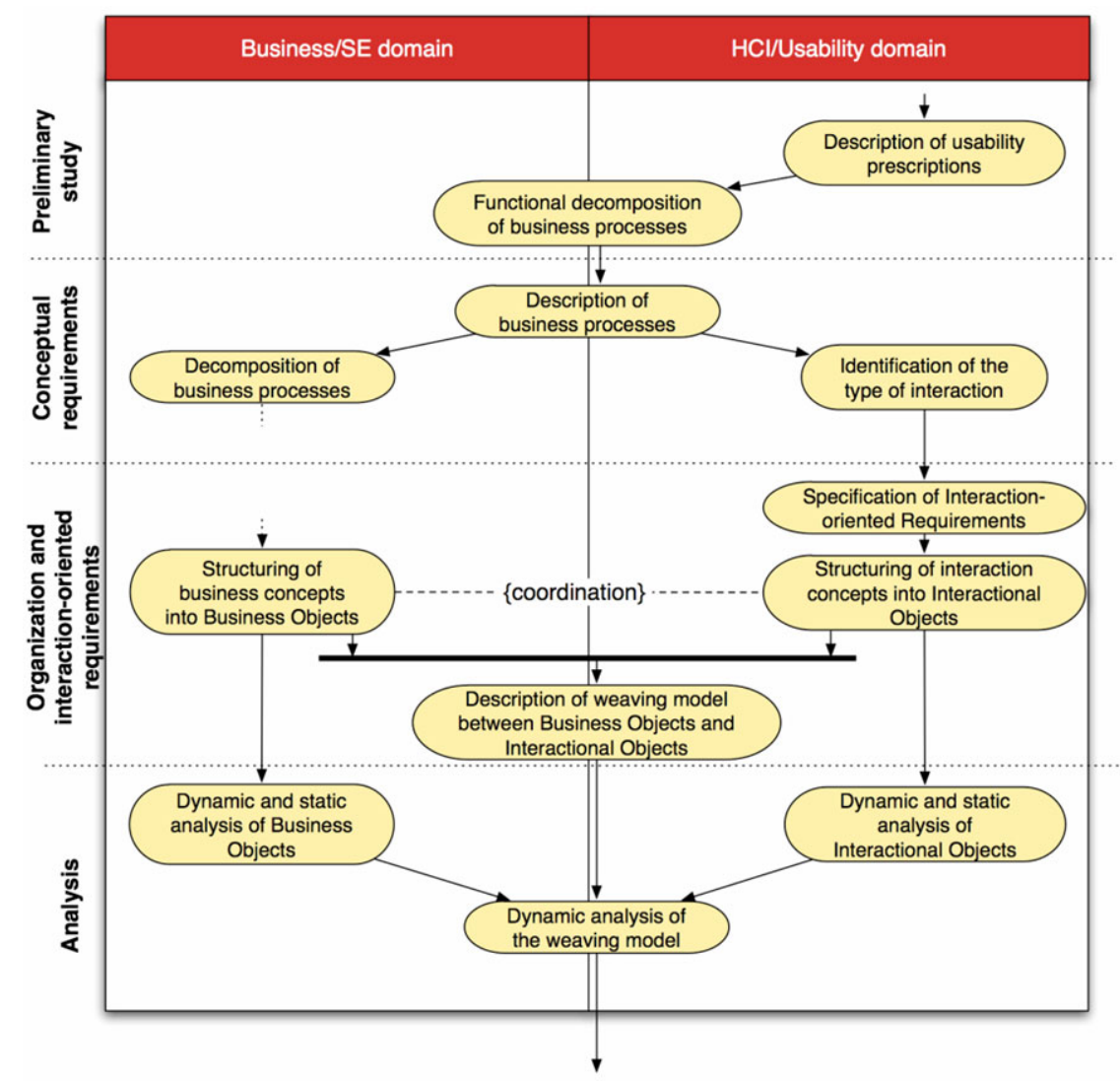

Fig. 1. Collaborations during the functional branch

The process starts with a cooperation study phase whose goal is providing a common decomposition of business processes. Then for each business process scenarii are described to start with a common view of the application. Then each specialist works in parallel with her own models: for HCI, task trees [3] and interaction model [4] for rich user interfaces; use cases, sequences diagrams for SE. From these models, the SE and HCI specialists must structure their concepts using Symphony Objects that are reusable components. The interaction space is structured with Interactional Objects (IO [5]), which are user interface-oriented components. The business is designed into Business Objects (BO).

Then the SE and HCI experts identify whether they need to modify their models to facilitate their ulterior weaving. It is a coordination activity: the experts do not need to produce a common product; they compare their models to validate their design choices.

The organizational and interaction-oriented requirements phase ends with a cooperation where the design actors must work together to produce a common product. The "Description of weaving model between BO and IO" allows both the HCI and SE experts identifying which IO correspond to projections of BO. 
Finally the functional branch terminates by an analysis phase where the models are completed by refining the weaving model. It is not studied here, as it is only a refinement of the previous phases.

\section{Case Study}

\subsection{Setting of the Case Study}

Rather than considering the process performance, we focus on "what are the right things to do" for designing a system with a rich user interface. We choose a qualitative approach to gather an in-depth understanding of the subject studied, with smaller but focused samples. With this approach, a comparison with another method mixing HCI and SE practices [6,7] would be useful; but it would be difficult to realize as many variables need to be controlled to obtain a useful experiment.

The experimental design is inspired from the social probes [8], translated to the professional context. It is based on the use of treatment groups only (no control group) and on a qualitative collection of data.

Participants. Four groups of two designers were asked to specify the same system. They are members of research groups specialized in HCI and SE, with more or less the same profile. They are colleagues of the experimenters. None of them was introduced to the Extended Symphony Method beforehand and they were volunteers to use it.

Steps. All the groups worked on the same example (a collaborative tool for designing public spaces). They followed mainly five steps during one week: 1) The first step is the starting session: a questionnaire about work habits, a short introduction to the Extended Symphony Method and the example were given to the participants. 2) Each group had to work on the example. Participants worked along or with the specialist of the other domain. Each time that a designer worked, he recorded his results, the time spent, his goals, his difficulties in a form, giving us so a probe. 3) After four days, each group had to realize the cooperative activity "Description of weaving model between BO and IO" (Fig. 1) to link the HCI and SE models. This intermediary session was recorded to evaluate whether the HCI and SE models were difficult to merge. 4) The groups had other working sessions to finalize their proposal. 5) They presented their results and during a focus group session, they gave their opinion on the process. One of the participants was absent at this last session.

\subsection{Results}

The method was perceived as interesting and satisfying. Collaboration was mainly perceived as useful (5/7). It was cited as one of the elements to reduce errors thanks to a better understanding between people of different domains. One positive aspect for collaboration is the separation of concerns between SE and HCI. But the participants also appreciated the common vision, facilitated by the use of common models. Nevertheless one of HCI participants pointed out the necessity of a common approach between the two specialists. 
Globally the sequencing of activities was considered logical and natural. However three participants thought that the process can be long even if for the majority of the participants (6/7), it can make the system design more efficient. The main reasons given by the participants were: 1) the collaborators start the design with a shared vision (the initial scenario); 2) The designers must think at the appropriate level of abstraction according to the design process.

Regarding the efficiency, the duration of the project and of the collaborative exchanges varied a lot from one group to another. Two groups spent less than 4 hours of work while another one took four times more. One group spent less than $15 \%$ of its total work time on collaborative activities, while another group spent $85 \%$ of its total work time. In the second group, each specialist did not respect his role and most of the work was realized in cooperation.

From the model viewpoint, it has been pointed out that the process gave rise to too many models. It can become difficult to check their consistency. However in all the groups, scenarii were a reference for model consistency. Its use was perceived as facilitating cooperation at the beginning of the process. For the other models, amongst the four groups, two have not realized the weaving between Symphony Objects. But the HCI and the SE models were judged as consistent because the common concepts of the two domains were identified and named in the same way. For the groups that used Symphony Objects, we noted that some adjustments (addition/suppression of objects) were made to obtain consistent models during the intermediary session. The specialists (3/4) considered that Symphony Objects are "a bridge between SE and HCI, a good synchronization point".

\section{Evolution of the Extended Symphony Method}

As we mentioned previously, some drifts in the process were noticed: 1) the process can be too long. 2) Some collaborative activities can occur for inappropriate goals although they are not in the process.

The participants suggested us the following improvements: 3) the steps where the consistency between SE and HCI models must be checked must be more explicit. 4) The method should be adapted to the project size. 5) A glossary could be added to provide a clear and short description of concepts. 6) A description of the role of each specialist could help each one in understanding his role. 7) A more precise description of Symphony Objects could be provided.

The first evolution answers to the points 1 and 4 . The process was simplified. Many activities became optional. Only the activities that produce the essential models remain mandatory. These models are those used to communicate with the stakeholders (e.g. scenarii), or to concretize the collaboration (e.g. Symphony Objects model).

For points 2 and 3, we considered that our two types of collaborations were disturbing. There was a misunderstanding about the coordination notion. Now we only propose cooperative activities whose goal is clearer. The description of collaborative activities has also been enriched: each activity is now considered from the viewpoint of the responsibilities of each actor. This is also a partial answer to point 5 . 
Globally we improved the documentation to respond to the three last points: a definition of a term is given when using it (point 5); we added a description of each role at the beginning of the method description (point 6); we tried to be more precise about the level of abstraction expected for each model (point 7). We systematically introduced examples of the expected products in the description of an activity.

\section{Conclusion}

This paper describes a case study that gave us some insights about the Extended Symphony collaborative process. Even if this case study has no statistical value, it was interesting in a qualitative approach to gather a variety of feedbacks. It allowed us improving the process by simplifying it. Of course these improvements would need to be evaluated by some experiments. More generally, we argue for a more systematic use of the qualitative approach for method engineering. With this goal, we are currently working with evaluation specialists to describe some of their knowledge in a reusable manner.

\section{References}

1. Dupuy-Chessa, S., Godet-Bar, G., Pérez-Medina, J.-L., Rieu, D., Juras, D.: A Software Engineering Method for the Design of Mixed Reality Systems. In: Engineering of Mixed Reality, ch. 15. Springer, Heidelberg (2009)

2. Ceret, E., Dupuy-Chessa, S., Godet-Bar, G.: Using Software Metrics in the Evaluation of a Conceptual Component. In: 4th Int. Conf. On Research Challenge in Information Science RCIS 2010, Nice, France (2010)

3. Paterno, F.: ConcurTaskTrees: An Engineered Notation for Task Models. In: The Handbook of Task Analysis for Human-Computer Interaction, pp. 483-503. Lawrence Erlbaum Associates, Mahwah (2003)

4. Dubois, E., Gray, P., Nigay, L.: ASUR++: a Design Notation for Mobile Mixed Systems. Interacting With Computers 15(4), 497-520 (2003)

5. Godet-Bar, G., Rieu, D., Dupuy-Chessa, S., Juras, D.: Interactional Objects: HCI concerns in the analysis phase of the Symphony method. In: 9th International Conference on Enterprise Information Systems ICEIS 2007, Funchal, Madeira, pp. 37-44 (2007)

6. Fox, D., Sillito, J., Maurer, F.: Agile methods and User-Centered Design: How these two methodologies are being successfully integrated in industry. In: Proceedings of Agile 2008, pp. 63-72. IEEE Computer Society, Washington, DC, USA (2008)

7. Corlett, D.: Design: innovating with OVID. Interactions 7(4), 19-26 (2000)

8. Bernhaupt, R.: Usability and User Experience Evaluation in Non-Traditional Environments, HDR de l'Université Paul Sabatier, Toulouse (2009) 